\title{
Les costumes du sud de la Laponie
}

Organisation et désorganisation d'un système symbolique

Southern Lapland Dress: the Organisation et Desorganisation of a Symbolic

System

\section{Yves Delaporte}

\section{OpenEdition \\ Journals}

Édition électronique

URL : https://journals.openedition.org/tc/5024

DOI : $10.4000 /$ tc. 5024

ISSN : 1952-420X

Éditeur

Éditions de l'EHESS

\section{Édition imprimée}

Date de publication : 30 juin 2010

Pagination : 631-645

ISSN : 0248-6016

Référence électronique

Yves Delaporte, "Les costumes du sud de la Laponie », Techniques \& Culture [En ligne], 54-55 | 2010, mis en ligne le 30 juin 2013, consulté le 29 septembre 2022. URL : http://journals.openedition.org/tc/ 5024 ; DOI : https://doi.org/10.4000/tc.5024 


\section{LES COSTUMES DU SUD DE LA LAPONIE}

\section{Organisation et désorganisation d'un système symbolique}

in Techniques et culture 12, $1988: 1-19$

Aucun des travaux scandinaves consacrés au vêtement lapon (recensés dans Delaporte 1980) n'évoque une éventuelle fonction sémiotique. Il y a là de quoi surprendre, quand on connaît l'importance de cette fonction et l'extraordinaire diversité de ses manifestations dans les costumes populaires européens. À l'occasion d'enquêtes chez les Lapons du Nord de la Norvège et de la Finlande, j'ai bien découvert l'existence d'un système de signes, informant du statut de personne célibataire ou mariée (Delaporte 1979) ; cependant, cantonné à une seule commune, celle de Kautokeino, porté par une seule pièce, la ceinture, et limité aux seuls jours de fête, ce système paraissait ne constituer qu'une exception ne remettant pas en cause le caractère généralement non signifiant des costumes lapons - au point que j'ai pu parler à leur propos de « degré zéro du signe » (Delaporte 1981).

Or lorsque mon enquête, qui visait à une étude exhaustive des quelque vingt costumes régionaux lapons, m’a conduit dans les territoires du Sud de la Laponie (provinces norvégiennes du Nordland, du Nord-Tröndelag et du Sör-Tröndelag, provinces suédoises du Västerbotten, du Jämtland et du Härjedalen), j'ai pu réunir un ensemble de faits prouvant l'existence d'un système de signes très élaboré. Ensemble à vrai dire disparate, ce système ne survivant à peu près intact que dans une seule et minuscule localité (hameau de Majavatn, dans la commune de Grane, Nordland), et étant partout ailleurs réduit à l'état de traces. Celles-ci sont cependant apparues suffisamment nombreuses et cohérentes pour que puisse être affirmée l'ancienne extension, à l'ensemble des six provinces en question, d'un système analogue, à quelques variations régionales près, à celui observé à Majavatn. 
C'est ce système que je me propose d'analyser ici, en empruntant à la sémiologie ${ }^{1}$ quelques-uns de ses outils conceptuels. Loin d'être un pur formalisme, une telle analyse, qui s’appuie sur la généralisation du modèle linguistique, permettra au contraire de fournir une explication de l'état actuel de ce système, et notamment de comprendre pourquoi certains de ses éléments survivent mieux que d'autres.

Dans la vaste région qui fait l'objet de cette étude, la minorité lapone est très dispersée, et en partie mêlée, sinon assimilée, à la population scandinave. Contrairement à ce qui se passe plus au nord, le costume traditionnel est depuis longtemps abandonné comme habit quotidien, et bien souvent seules quelques pièces sont encore portées lors des jours de fête. L'aspect signifiant de ces pièces est encore conscient à Majavatn et dans ses environs immédiats ; ailleurs il a en grande partie disparu, et l'enquête orale doit donc souvent laisser la place à la seule observation directe des pièces encore portées occasionnellement, ou de celles qui, n'étant plus portées, ont cependant été conservées dans quelque réserve. Cette opposition entre deux régions, l'une où le système présente une organisation cohérente, l'autre où le processus de désorganisation est largement avancé, guidera l'exposé des faits.

\section{Le système organisé}

\section{Symbolisme des couleurs}

À Majavatn, une relation de type symbolique ${ }^{2}$ est établie entre les couleurs utilisées pour orner le costume, les éléments naturels et les deux sexes : le jaune est la couleur du soleil ; le bleu, couleur de l'eau, est "porté par l'homme »; le rouge, couleur du feu, est " porté par la femme ». La tradition orale rapporte que la liaison du jaune avec le soleil, principale divinité de l'ancienne religion lapone, a entraîné une moindre utilisation de cette couleur depuis l'époque de la christianisation : les missionnaires auraient combattu son emploi dans le costume, faisant pression sur les marchands pour qu'ils cessent de vendre du drap jaune ; en même temps, ils auraient cherché à imposer la couleur verte, couleur composite qui, selon mes informateurs, n'appartiendrait pas au costume lapon « authentique ».

\section{Signifiés 'homme' et 'femme'}

La répartition du bleu et du rouge dans le vêtement des adultes s'organise autour de deux principes. En premier lieu, chacune de ces couleurs, si elle n'est pas portée exclusivement par le sexe auquel elle est liée, domine dans l'ornementation de son costume. En second lieu, les positions respectives du bleu et du rouge sont régies par un principe d'inversion entre les costumes masculin et féminin. Ces deux principes sont mis simultanément en œuvre dans les coiffures et les plastrons, tandis que le second se réalise seul dans les tuniques et les ceintures (figure 1):

a) La coiffure est bleue avec incrustations rouges chez l'homme, rouge avec applications bleues chez la femme.

b) Le plastron est bleu avec applications rouges chez l'homme, rouge avec 
applications bleues chez la femme. La broderie d'étain qui orne cette pièce n'y a qu'une fonction esthétique.

c) Dans la tunique, les poignets et la fente d'ouverture sont ornés sur leurs bords (c'est-à-dire en position que nous conviendrons d'appeler externe) d'une bande bleue chez l'homme, rouge chez la femme; et, en position interne, d'une bande rouge chez l'homme, bleue chez la femme. Ces deux bandes sont séparées du drap bleu de la tunique par une troisième, de couleur jaune. Lordonnance des couleurs dans le bas de la tunique féminine est identique à celle des poignets : rouge externe, bleu interne ; mais il n'y a pas sur ce point d'opposition avec la tunique masculine, celle-ci ne comportant pas d'autre ornementation dans le bas qu'un mince liseré blanc.
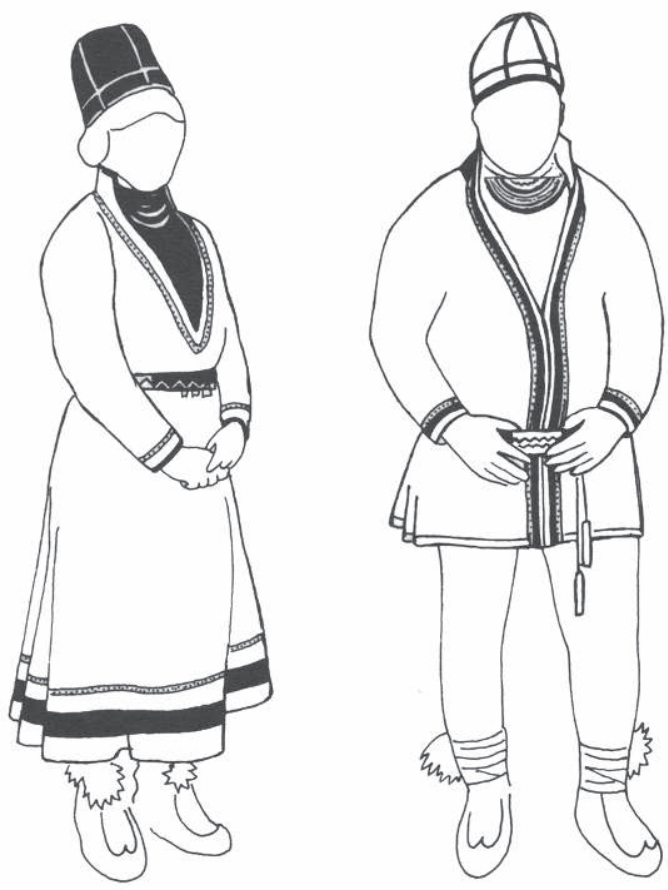

Costumes de femme et d'homme adultes (Majavatn) (Fig. 1)

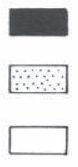

rouge

jaune

bleu (pour les tuniques, coiffures, plastrons et ceintures seulement; cette convention vaut également pour les fig. 3 et 4 )

La couleur bleue du drap utilisé ici, comme dans toute la Laponie, pour fabriquer les tuniques des deux sexes, ne constitue pas un élément pertinent du code. Or, la bande rouge qui orne le bas de la tunique féminine n'est pas située à son bord, mais un peu plus haut : il s'ensuit qu'elle est intercalée entre deux bandes de couleur bleue, dont l'une est sémiotiquement pertinente et l'autre non. Cette ambiguité du bleu brouille singulièrement la lecture, et constitue l'une des causes des difficultés d'interprétation auxquelles nous 
nous heurterons dans les autres régions. S'agissant des tuniques portées à Majavatn, les deux bandes liées aux signifiés 'homme' et 'femme' doivent être comptées à partir de la bande jaune : celle-ci, qui n'est en rien associée par elle-même à ces signifiés, joue donc un rôle d'indicateur de pertinence. Ce problème ne se pose pas dans le cas des variantes individuelles, rencontrées hors de Majavatn, qui remplacent les bandes décoratives bleues par des bandes vertes, et c'est manifestement pourquoi la bande jaune est alors le plus souvent absente.

d) Un premier modèle de ceinture, le plus ancien, est divisé en deux par un axe longitudinal délimitant une moitié à dominante rouge et une moitié à dominante bleue. Un second modèle, plus récent, présente une alternance de segments rouges et bleus, au nombre de six : il y a donc une extrémité rouge et une extrémité bleue. Ces ceintures (figure 2) sont identiques pour les deux sexes, mais portées différemment : le modèle ancien est porté par l'homme de manière que le bleu soit en bas, et le modèle récent de manière que l'extrémité bleue, venant recouvrir l'extrémité rouge, soit seule visible. La femme dispose sa ceinture en sens inverse : s'il s'agit du modèle ancien, la partie rouge est en bas, et s'il s'agit du modèle récent, l'extrémité rouge vient recouvrir l'extrémité bleue. En outre ces deux modèles se nouent en principe à gauche chez la femme, à droite chez l'homme.

\footnotetext{
Ceintures (Majavatn)

En haut : modèle ancien ;

en bas : modèle récent.

(Fig. 2)
}

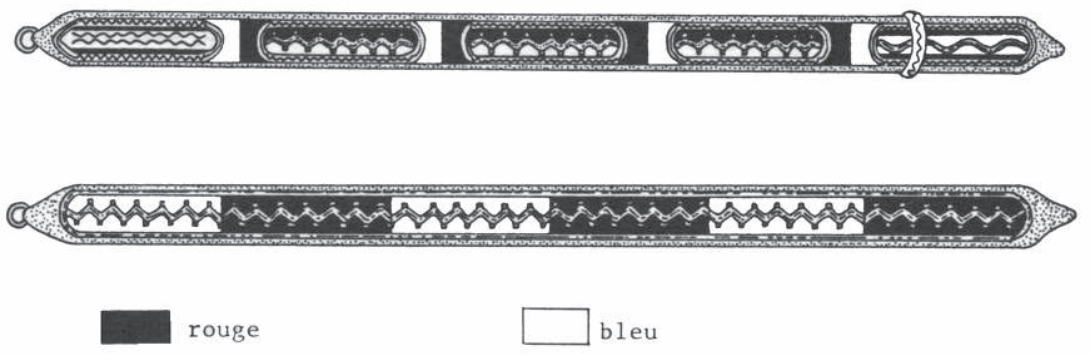

Selon les pièces qu'elles investissent, les couleurs ont des statuts sémiotiques quelque peu différents. Dans les tuniques et les ceintures, les unités signifiantes sont constituées par l'association du bleu, du rouge, et de leur position respective. Elles sont donc analysables en unités plus petites, les couleurs, qui, n'étant pas elles-mêmes associées ici à un quelconque signifié, ne sont pas des signifiants et ont donc valeur d'unités de seconde articulation ${ }^{4}$. L'ordonnance de ces unités s'inversant entre les pièces masculines et féminines, les signes correspondants apparaissent fondés sur une motivation de type homologique : les signifiants sont dans le même rapport inversé que les signifiés.

Le cas des coiffures et des plastrons est plus ambigu : si la production du sens s'y organise de manière formellement identique au cas précédent, la prédominance du bleu dans la coiffure et le plastron masculins, ainsi que du rouge dans la coiffure et le plastron féminins, indique que ces couleurs gardent en outre un lien direct avec leurs significations propres, et qu'elles fonctionnent partiellement comme des unités de première articulation, c'est-à-dire des signes possédant une face signifiante et une face 
signifiée $(/ \mathrm{bleu} /=$ 'masculin',/rouge/ = 'féminin'). Dans ces deux pièces, les couleurs ont donc un statut intermédiaire entre ceux d'unités de première et de seconde articulation ; ce cas n'est pas sans évoquer, mutatis mutandis, la question depuis longtemps débattue de la valeur expressive des phonèmes, les unités linguistiques de seconde articulation.

e) Les bandes tissées que l'on enroule autour de la haute tige des mocassins constituent un dernier exemple de répartition des couleurs différant selon le sexe, mais cette répartition n'apparaît plus fondée sur une motivation analogique (le bleu et le rouge sont en proportions égales) ni même homologique (on ne peut transformer l'un des signifiants en l'autre par inversion). Chez la femme, cette pièce est composée de trois bandes de couleur unie, rouge, bleu (ou vert) et jaune, assemblées bout à bout et prolongées par des tresses et des pompons où les couleurs restent séparées. Chez l'homme, elle forme un écossais rouge, bleu (ou vert) et jaune, prolongé par des tresses et des pompons où les couleurs sont mêlées.

\section{Signifiés 'veuvage' et 'deuil'}

La seconde opposition traduite par le vêtement est celle qui s'établit entre les personnes mariées dont le conjoint est vivant, et celles dont le conjoint est décédé. Elle est fondée sur le principe suivant : « L'homme prend la couleur de la femme, la femme prend la couleur de l'homme». Pendant l'année qui suit le décès, le veuf ou la veuve portent en effet la coiffure, le plastron, les bandes d'attache des mocassins qui appartenaient à leur conjoint. Chacun garde sa propre ceinture, mais la porte à l'envers, à la manière de l'autre sexe. En outre, la veuve inverse l'ordre des couleurs dans l'ornementation de sa tunique, adoptant donc celle qui caractérise la tunique masculine.

Lannée écoulée, le veuf et la veuve reprennent leur propre costume. Mais pour le reste de sa vie, la veuve conserve l'ordre inversé des couleurs de sa tunique (figure 3). Cette inversion concerne non seulement l'ornementation des poignets et de la fente d'ouverture, mais également celle du bas de la tunique. Ce dernier détail est important pour l'analyse du système : il confirme en effet que, dans l'opposition 'homme' et 'femme', l'ordonnance des couleurs du bas de la tunique féminine doit être regardée comme pertinente, bien que, considérée isolément, elle n'entre pas en opposition avec une ornementation correspondante de la tunique masculine.
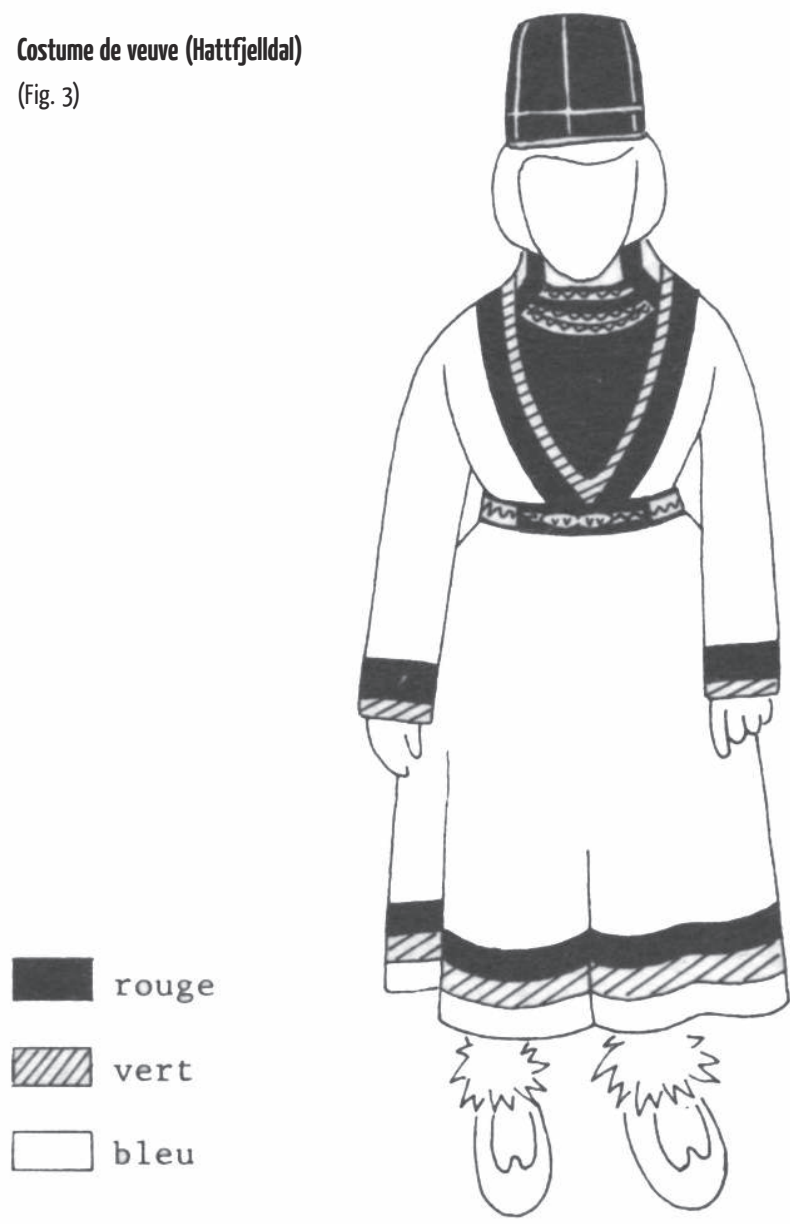
Enfin, pour annoncer le deuil d'un parent qui n'est pas le conjoint, l'homme ou la femme portent leur propre tunique à l'envers, c'est-à-dire retournée de manière à ce que les coutures soient visibles. Usage illustré par ce commentaire d'un informateur : « Je suis avec mon frère dans la montagne, et il meurt à la suite d'un accident. Alors, pour redescendre au village, je retourne ma tunique. Je n'ai pas besoin de parler, dès que les gens me voient, ils comprennent ce qui s'est passé. » Selon cet informateur, la fonction d'un tel usage serait de permettre de garder le silence au cours de cette terrible épreuve, alors que l'on est écrasé par la douleur : « Le costume remplace le langage», affirme-t-il.

Costume de fillette (Majavatn) (Fig. 4)

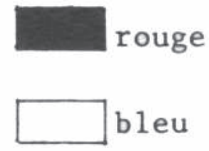

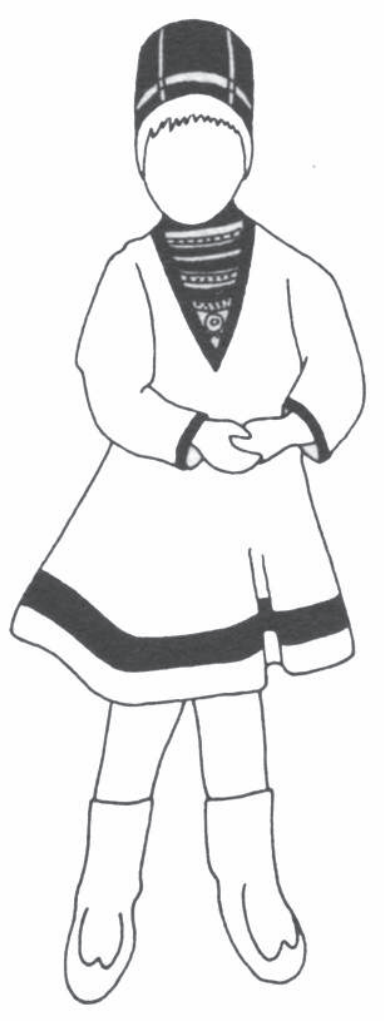

\section{Signifié 'âge'}

Les coiffures et plastrons portés par les enfants sont identiques à ceux des adultes : leurs différences ne sont donc pertinentes que par rapport à l'opposition des sexes. Les tuniques ont en revanche une ornementation simplifiée par rapport à celles portées par l'homme et la femme (figure 4), le changement s'effectuant à l'époque de la communion solennelle, vers quatorze ans. Avant cet âge, les enfants ne portent sur leur tunique que la couleur qui est associée à leur sexe (bandes bleues pour les garçons, rouges pour les filles), le passage à l'âge adulte entraînant l'ajout, selon les modalités qui ont été décrites plus haut, de la couleur de l'autre sexe. Chez les enfants, les couleurs des bandes décoratives ont donc pleine valeur d'unités de première articulation, fondées sur une motivation de type analogique.

\section{Signifiés 'jeune fille' et 'femme mariée'}

Le costume de femme mariée se distingue de celui de jeune fille par la présence d'un col droit dans la tunique (figure 1), un élément habituellement réservé, dans tout le reste de la Laponie, aux seules tuniques masculines.

Le port de bandes bleues, qui traduit le passage du statut de fillette à celui de jeune fille, puis le port d'un col droit, qui traduit le passage du statut de jeune fille à celui de femme mariée, enfin l'inversion des bandes décoratives, qui traduit le passage du statut de femme mariée à celui de veuve, sont donc trois manifestations d'une même tendance à valeur symbolique, consistant en l'ajout progressif, au cours de la vie d'une femme, d'éléments masculins dans sa tunique.

Une construction aussi sophistiquée pose deux problèmes. En premier lieu, il apparaît peu vraisemblable qu'il s'agisse là d'un symbolisme lié au seul vêtement; on serait plutôt tenté d'y voir le reflet d'une pensée symbolique plus générale concernant les catégories du masculin et du féminin, et dont, peut-être, ne subsiste aujourd'hui que la seule traduction vestimentaire. En second lieu, le lien évident qui existe, chez les Lapons du Nord, entre différents traits de leur culture et le caractère peu signifiant de leurs costumes 
(Delaporte 1981) conduit à s'interroger sur les traits culturels spécifiques produisant, chez les Lapons du Sud, un tel symbolisme. Labsence de travaux ethnologiques sur la culture des Lapons du Sud (ma propre enquête n'ayant été qu'extensive) oblige à laisser ces deux questions en suspens.

\section{Le système désorganisé}

\section{Symbolisme des couleurs}

Sur l'existence d'associations entre couleurs et éléments naturels, et leurs conséquences sur le port du jaune et du vert, je n'ai pu obtenir de renseignements ailleurs qu'à Majavatn. Cependant, il est patent que partout dans le sud, l'emploi du jaune dans l'ornementation de la tunique est très réduit. Utilisée abondamment dans les communes jouxtant au nord la région étudiée, cette couleur est à peu près absente de toutes les tuniques du Västerbotten, du Jämtland, du Härjedalen et du Sör-Tröndelag. Lorsqu'elle apparaît dans une minorité de tuniques du Nordland et du Nord-Tröndelag, c'est toujours avec modération : les bandes jaunes sont constamment d'une largeur moindre (souvent la moitié) que celle des bandes rouges, bleues ou vertes ; à Snåsa, l'abus d'ornementation jaune est regardé comme une preuve de vanité.

Quant au vert, très utilisé hors de Majavatn, y compris dans les hameaux voisins, il possède un statut sémiotique bien particulier : il apparaît rigoureusement interchangeable avec le bleu. Occupant toujours une position identique dans l'alternance des bandes décoratives de chaque costume régional, et n'apparaissant que très rarement ensemble dans l'ornementation d'une même pièce, ces deux couleurs peuvent être regardées comme synonymes, le choix de l'une ou l'autre relevant de la seule variabilité individuelle. Cette synonymie suggère la possibilité d'un emprunt tardif de l'une de ces deux couleurs, et me paraît donc pouvoir être retenue en faveur de l'authenticité de la tradition orale recueillie à Majavatn.

S'agissant enfin du lien entre couleur et sexe, son caractère symbolique, qui, comme on le verra, se maintient partiellement dans les faits, semble atténué dans la conscience des sujets : si on voit encore ici ou là dans le rouge et le bleu les couleurs respectives de la femme et de l'homme, c'est moins comme un principe fondateur du système que comme simple conséquence d'un état de fait, la prédominance du rouge dans le costume féminin et du bleu dans le costume masculin.

\section{Signifiés 'masculin' et 'féminin'}

a) Partout où sont portées des coiffures coniques relevant du même type que celles de Majavatn, elles sont bleues avec incrustations rouges chez l'homme, rouges avec applications (ou incrustations) bleues ou vertes chez la femme. Une coiffure féminine entièrement différente, portée comme alternative aux précédentes dans le Sör-Tröndelag et le Härjedalen, échappe à ce symbolisme (cf. infra) ; celui-ci se laisse en revanche deviner 
dans une coiffure masculine portée dans le Nord-Tröndelag, le Jämtland et le Härjedalen : de même type que celle portée à Majavatn mais plus évoluée (ajout de rabats et d'un gros pompon de laine rouge), elle est en drap bleu ou noir avec incrustations rouges.

Dans le Västerbotten et le Jämtland, j'ai rencontré deux modèles de coiffures portées spécifiquement par les enfants, où s'observe l'opposition du bleu (ou du vert) et du rouge. Ces coiffures sont donc liées à deux signifiés : 'sexe' et 'âge'. Comme on le verra à la lecture des descriptions qui suivent, il n'y a pas amalgame des signifiants, chacun de ces deux signifiés pouvant être mis en relation avec des éléments distincts : la coupe signale l'appartenance à une classe d'âge, celle de l'enfance, tandis que la répartition des couleurs traduit le sexe du porteur.

Un modèle ancien, qui n'est plus porté aujourd'hui, recouvrait les oreilles et la nuque, et se caractérisait par la présence, à son sommet, d'une pièce ronde. Chez la fillette, cette coiffure était rouge avec un sommet bleu; chez le garçon, elle était bleue avec un sommet et un bord rouges (commune de Sorsele, Västerbotten). Deux coiffures de forme voisine, provenant de la commune de Frostviken (Jämtland) et accompagnées de la mention « bonnets d'enfants » figurent dans les collections du Nordiska Museet (Stockholm) : on peut aisément les identifier comme étant respectivement une coiffure de fillette, rouge avec ornementation verte, et une coiffure de garçon, bleue avec ornementation rouge.

Un modèle plus récent (village de Jänsmässholmen, Jämtland) est une variante de celui qui est porté à Majavatn : il comprend comme lui six pièces triangulaires, mais les pièces latérales viennent couvrir les oreilles, et il y a ajout d'un gros pompon. La coiffure de fillette est en drap rouge avec incrustations passepoilées vertes et cordons d'attache rouges, celle du garçonnet est en drap bleu avec incrustations passe-poilées rouges et cordons bleus. Les pompons sont en laine rouge et verte, avec un peu de jaune dans celui de la coiffure de garçon.

Les signes portés par ces coiffures pour enfants sont, comme à Majavatn, fondés sur une motivation à la fois homologique et analogique, jouant simultanément sur une inversion dans la répartition des couleurs entre sexes, et une dominance de la couleur associée à chaque sexe. Cette parenté dans l'organisation sémiotique est d'autant plus frappante qu'elle concerne des pièces de coupe et d'ornementation différentes.

b) Dans les six provinces étudiées, les plastrons sont presque toujours à dominante bleue chez l'homme, rouge chez la femme. Cependant, certains plastrons portés dans le Sör-Tröndelag et le Härjedalen sont trop ornés pour que l'on puisse leur attribuer aisément une couleur dominante.

c) En ce qui concerne les tuniques, il est beaucoup plus difficile d'aboutir à des conclusions tranchées. On se heurte en effet à de multiples difficultés : le trop petit nombre de tuniques masculines encore observables, qui rend difficile une comparaison systématique avec les tuniques féminines ; l'existence d'une variabilité individuelle importante ; l'ajout fréquent, à des fins purement esthétiques, de bandes décoratives autour de la fente d'ouverture des tuniques féminines, empêchant l'identification de celles qui pourraient avoir une fonction sémiotique; l'inversion des couleurs à mi-hauteur de la fente d'ouverture des tuniques masculines (dans le Sör-Tröndelag et le Härjedalen, assez souvent également dans le Nord-Tröndelag), qui rend sans objet la confrontation avec l'ordonnance des couleurs dans les tuniques féminines; le rôle équivoque du bleu, dont on ne sait parfois, particulièrement lorsqu'il borde la fente d'ouverture, s'il faut le rattacher au fond de la tunique ou le considérer comme une bande décorative. 
L'enquête orale est ici totalement décevante, toute trace d'une éventuelle signification de l'ordonnance des couleurs dans les tuniques étant absente des représentations ${ }^{5}$. J'ai donc cherché, en procédant à des comptages, à tester l'hypothèse de la persistance d'une répartition signifiante des couleurs, forme qui aurait pu se transmettre par tradition tout en se vidant de son contenu originel. Dans les trois provinces norvégiennes (Nordland, Nord-Tröndelag, Sör-Tröndelag), il est apparu que les tuniques observées tendaient majoritairement à respecter le système en vigueur à Majavatn. En revanche, dans les trois provinces suédoises (Västerbotten, Jämtland, Härjedalen), la grande variabilité de l'ornementation me paraît exclure toute survivance, même purement formelle, de ce système : la répartition des couleurs y semble aléatoire, due à la seule fantaisie des individus ou soumise à des modes locales.

d) Le cas des ceintures se complique du fait que les éléments signifiants appartiennent à deux catégories différentes : aspect de la pièce d'une part, manière de la porter d'autre part. Il faut donc, en face d'une ceinture, non seulement examiner si son aspect est tel qu'il puisse se prêter à un emploi signifiant (par exemple alternance de segments rouges et bleus en nombre pair), mais également s'assurer qu'elle est ou a été portée de manière adéquate. Comme dans le cas des tuniques, on doit envisager un maintien de la forme allant de pair avec une disparition du contenu.

Le modèle de ceinture comprenant des segments alternés de couleurs opposées (rouge vs bleu ou vert) se rencontre dans le Nord-Tröndelag et le Västerbotten, en particulier à Tärna et Vilhelmina; mais ce modèle ne semble pas être porté de manière à traduire l'opposition des sexes. Dans ces deux communes, j'ai également rencontré trois nouveaux modèles de ceintures, où les modes de répartition des couleurs, pour être différents de ceux observés précédemment, n'en ont pas moins un contenu manifestement symbolique. Un modèle de ceinture féminine se caractérise par une bande centrale verte et des bandes latérales rouges. Un premier modèle de ceinture masculine présente une répartition inverse des couleurs : bande centrale rouge, bandes latérales bleues ; on est clairement ici en présence d'une motivation de type homologique. Un second modèle de ceinture masculine qui, en revanche, ne comporte que du bleu, correspond à une motivation de type analogique.

e) Les bandes d'attache des mocassins offrent le plus souvent une répartition des couleurs identique à celle observée à Majavatn, le bleu étant toutefois partout supplanté par le vert.

f) Enfin, dans la commune de Vilhelmina, le code investit une nouvelle pièce, les chaussures (en fourrure de renne pour l'hiver, portées par les adultes; en cuir de renne pour l'été, portées par les enfants) : les incrustations de drap de couleur dans la couture d'assemblage de l'empeigne avec les parties latérales sont bleues pour le sexe masculin, rouges pour le sexe féminin.

\section{Signifiés 'veuvage’et 'deuil'}

Hors de Majavatn ou de ses environs immédiats, les deux seuls éléments encore vivants que j’ai rencontrés sont le port de la coiffure du conjoint décédé (Jänsmässholmen) et l'inversion des couleurs dans la tunique de veuve (Snåsa). N'appartiennent plus qu'à la tradition orale : le port de la coiffure et des bandes d'attache des mocassins du conjoint (Snåsa, Tärna, Saxnäs, Vilhelmina), de son plastron (Snåsa, Tärna), de sa 
ceinture (Tärna, Saxnäs), ainsi que l'inversion des couleurs dans la tunique de veuve et le port inversé de la tunique pour annoncer un deuil (Tärna, Saxnäs).

Que les signes de veuvage se maintiennent dans les faits ou ne soient plus qu'un souvenir, ils sont toujours réduits à l'état d'éléments isolés et perdent donc une grande partie de la valeur symbolique qui est la leur à Majavatn. Dans cette localité, les modes de répartition des couleurs dans le costume de l'homme, dans celui de la femme mariée, dans celui de la veuve, forment en effet une série dans laquelle chaque terme est obtenu en inversant le terme précédent : l'habillement de la veuve y est donc pensé comme l'inversion d'une inversion. Ailleurs, cet habillement n'est, plus prosaiquement, regardé que comme une coutume selon laquelle la veuve doit porter certaines pièces du costume de son mari.

\section{Signifié ‘âge'}

L'emploi, dans la tunique de fillette, de bandes décoratives exclusivement rouges se retrouve au hameau de Sefrivatn (Nordland), tandis que la tunique de garçonnet y est en tous points identique à celle de l'homme adulte. Là encore, la désorganisation du système modifie la valeur des éléments qui se maintiennent. À Majavatn, l'ornementation de la tunique de fillette (bandes rouges externes) l'oppose à celle du garçonnet (bandes bleues externes), de la femme (bandes rouges externes, bleues internes) et de l'homme (bandes bleues externes, rouges internes): on peut donc l'analyser comme étant constituée d'un signifiant/bandes d'une seule couleur/ qui renvoie au signifié 'enfant', et d'un signifiant/rouge externe/ qui renvoie au signifié 'de sexe féminin'. À Sefrivatn, où il y a disparition de l'opposition entre enfant et adulte dans les tuniques masculines, l'ornementation sert toujours de signifiant au signifié 'fillette', mais c'est alors un signifiant amalgamé, dans lequel il n'est plus possible de distinguer ce qui renvoie à l'âge, et ce qui renvoie au sexe.

Ailleurs, le signifié 'âge' est absent, ou traduit par de nouveaux signifiants : broderies de perles sur le plastron de fillette, broderie de fil d'étain sur celui de femme (Snåsa) ; bandes de couleur continue sur la fente d'ouverture de la tunique de garçonnet, alternance des couleurs à mi-hauteur sur la fente d'ouverture de la tunique d'homme (Snåsa) ; à Vilhelmina, la présence d'un col droit dans la tunique féminine n'oppose pas, comme à Majavatn, la jeune fille et la femme mariée, mais deux classes d'âge : la fillette d'une part, la jeune fille ou la femme mariée d'autre part. Également à Vilhelmina, la fillette porte des bandes d'attache pour mocassins en laine jaune et verte; après la communion solennelle, on y ajoute de la laine rouge. Par contre, ces bandes sont identiques chez le garçon et l'homme adulte.

\section{Signifiés 'jeune fille' et 'femme mariée'}

À Vilhelmina, l'ordonnance des couleurs dans la tunique féminine s'inverse après le mariage : rouge interne sur la fente d'ouverture, rouge au-dessus du vert dans le bas ; comme il est logique, l'inversion accompagnant le veuvage est ici inconnue. Cela, joint au fait que le port d'un col droit traduit le passage du statut de fillette à celui de jeune fille, conduit à reconnaître, sous une forme à la fois rudimentaire et modifiée, le principe symbolique en vigueur à Majavatn, consistant en l'ajout progressif d'éléments masculins dans la tunique féminine. 
La coiffure féminine d'une coupe spécifique que l'on rencontre dans le Härjedalen et le Sör-Tröndelag comme alternative à la coiffure conique est rouge et ornée de perles chez la jeune fille, bleue ou verte et ornée de fil d'étain chez la femme mariée. II y a là une combinaison originale d'éléments signifiants rencontrés ailleurs : on retrouve l'opposition entre perles et étain, associée à Snåsa au signifié 'âge'; ainsi que la tendance générale à utiliser dans le costume de la femme adulte ou mariée des éléments (ici, la couleur bleue) masculins.

\section{Mode d'organisation et destin du système}

L'ensemble des observations qui viennent d'être présentées prouve sans conteste un fait nouveau pour l'ethnologie lapone, l'existence d'un ancien système symbolique dans toute la Laponie du Sud. Au-delà de la distinction entre une aire extrêmement réduite où ce système se maintient dans les faits et les consciences, et une aire beaucoup plus vaste (figure 5) où ne peuvent être repérés que des éléments isolés, parfois détachés de toute représentation consciente, ce qui frappe l'observateur, c'est avant tout l'unité du phénomène, dont on ne peut trouver meilleure illustration que son investissement dans des pièces de coupes parfois fort différentes les unes des autres.

Bien mieux, le réexamen de toutes les coiffures portées dans l'ensemble de la Laponie, à la lumière des observations faites dans le sud, révèle une tendance générale à la prédominance du bleu chez l'homme, du rouge chez la femme. Ce fait, resté inaperçu jusqu'ici en raison de la diversité des formes, de la prolifération d'une ornementation à fonction purement esthétique, de phénomènes d'emprunt tardif, ainsi que de son absence dans les représentations, suggère que l'opposition principale qui fonde le système en vigueur à Majavatn pourrait avoir été répandue autrefois dans toute la Laponie.

Pour conclure, on voudrait attirer l'attention sur le lien qui existe entre l'état actuel de ce système et quelques-unes des caractéristiques mises en évidence par l'analyse sémiologique.

On observera tout d'abord que, dans la forme sous laquelle il se manifeste à Majavatn, le système est constitué par des signes dont la face signifiée est toujours associée à des signifiants discontinus c'est-à-dire portés par des pièces distinctes. Le signifié 'femme [non veuve]' par exemple, est traduit par le signifiant /coiffure rouge ornée de bleu/ + / plastron rouge orné de bleu/ + /tunique dont les poignets, la fente d'ouverture et le bas sont ornés de rouge en position externe, de bleu en position interne/ + /ceinture dont l'extrémité visible est rouge/. Or, cette discontinuité a une fonction de redondance : chaque pièce portant l'un des éléments du signifiant transmet, entièrement et sans ambiguité, le signifié correspondant. L'illustration à la fois la plus simple et la 


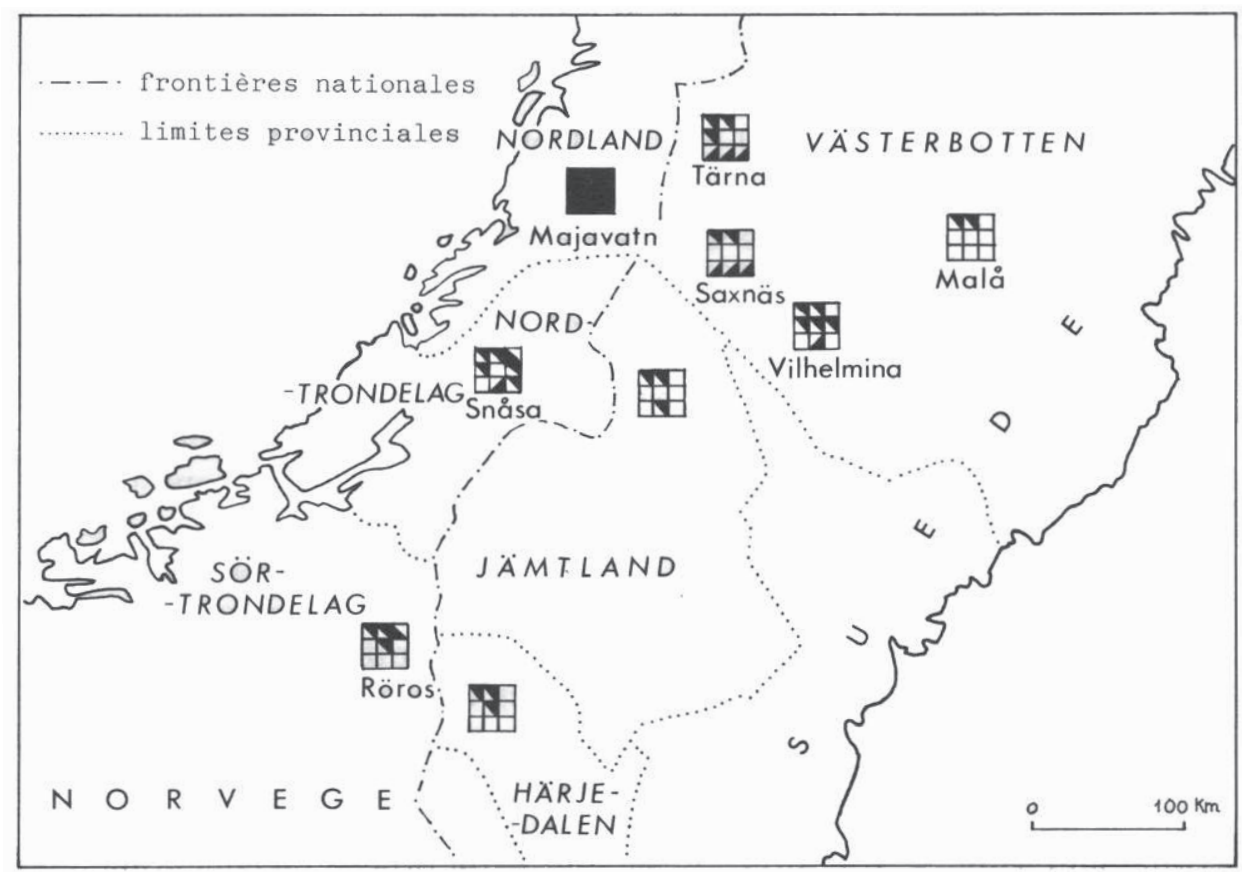

Symbolisme des costumes de Laponie méridionale : répartition des principaux cas

(Fig. 5)

米

Inversion des couleurs entre les coiffures coniques des deux sexes

H

Inversion des couleurs entre les plastrons des deux sexes

斗要

Inversion des couleurs entre les tuniques des deux sexes

Inversion des couleurs entre les ceintures des deux sexes

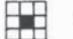

Distinction entre costumes de jeune fille et de femme mariée

P1

Distinction entre costumes portés avant et après la communion

Port de pièces inversées pour annoncer un deuil

巴

Port de pièces appartenant au conjoint décédé

曲

Inversion des couleurs dans la tunique de veuve

- Elément conservant aujourd'hui un contenu symbolique

- Elément se maintenant comme forme séparée de son contenu symbolique

v Elément se maintenant comme forme séparée de son contenu sémiotique

a Elément présent seulement dans la tradition orale

mieux connue d'une situation inverse se trouve dans le costume morave étudié par P. Bogatyrev (1971), où les signifiés associés à une certaine coiffure ('fiancé' ou 'conscrit') diffèrent selon qu'il y a absence ou présence d'une autre pièce, un certain modèle de pantalon en bure. 
Le système étant constitué d'éléments qui, du point de vue de la production du sens, peuvent être considérés comme indépendants ${ }^{6}$, il s'ensuit que certains d'entre eux peuvent céder sous la pression de l'acculturation sans que cela remette en cause le maintien des autres. Pour nous référer à nouveau au costume morave analysé par Bogatyrev, il est bien évident que le destin de la coiffure comme élément signifiant est lié à celui du pantalon de bure : que celui-ci devienne obsolète, et celle-là verra sa signification se modifier ou disparaître. Rien de tel ici, où le lien est manifeste entre la faible structuration du système et ce que celui-ci est devenu dans l'ensemble de la Laponie du Sud: état général de désorganisation qui correspond à la situation actuelle des Lapons du Sud, minorité submergée par la société moderne ; et, contrastant avec cet état dégradé, maintien surprenant de traits isolés. Au cours des différentes étapes du processus de désorganisation, le caractère faiblement structuré du système a d'ailleurs pu influer dans des sens opposés : si, au stade d'abandon du costume atteint aujourd'hui, on constate son influence conservatrice, il est vraisemblable qu'à un stade plus précoce il ait au contraire exercé une influence dissolvante.

Quant aux facteurs qui permettent de comprendre pourquoi ce sont telles parties du système qui ont disparu, et telles autres qui se sont maintenues, ils peuvent être trouvés dans la nature des éléments utilisés comme signifiants, ainsi que dans le type d'articulation et de motivation auxquels répondent les différents signes. S'agissant tout d'abord des catégories auxquelles appartiennent les éléments signifiants : parmi celles-ci, coupe, matériau, ornementation ou manière de porter une pièce, la dernière est la seule qui ne soit pas intégrée dans la pièce, mais qui soit liée à la permanence, dans la conscience des sujets, du symbolisme fondant le système ; cette catégorie de signifiant, à laquelle appartiennent ici les ceintures, est à l'évidence la plus labile. S'agissant ensuite du type d'articulation et de motivation : les éléments qui se maintiennent le mieux sont ceux que nous avons identifiés comme des unités de première articulation, fondés, au moins partiellement, sur une motivation de type analogique : pièces à dominante bleue ou rouge, en l'occurrence coiffure et plastron. Les éléments résultant de la combinaison d'unités de seconde articulation et se caractérisant par une motivation qui n'est qu'homologique - deux traits tendant concurremment ${ }^{7}$ à rendre plus lâche le lien entre signifiant et signifié - se montrent davantage vulnérables. Le cas de l'ornementation des tuniques est ici exemplaire : sa fonction sémiotique disparaît le plus souvent, brouillée par la fonction esthétique ; et, lorsqu'elle se maintient, c'est pour traduire de nouvelles oppositions, la motivation homologique se prêtant bien, dans le cadre de la variabilité régionale, à des transferts d'emploi.

Dans le cas des bandes d'attache des mocassins pour homme et pour femme, le lien entre signifiants et signifiés est particulièrement distendu, puisqu'il n'apparaît guère possible d'y reconnaître une motivation analogique ou homologique. Cependant, la répartition des couleurs dans ces deux pièces, loin de se montrer labile, est au contraire l'un des éléments qui résistent le mieux dans le cadre du système désorganisé. Cette apparente contradiction se résout si l'on observe que ce qui permet aux éléments signifiants de se maintenir n'est pas d'ordre sémiotique, mais technique. L'opposition entre les deux modes de répartition des couleurs est en effet indissolublement liée à la construction même des deux pièces en question; s'agissant de bandes tissées, ce lien est d'autant plus fort qu'elles appartiennent à la catégorie, mise en évidence par H. Balfet (1981), de celles qui sont construites par fabrication du matériau. 


\section{NOTES}

Toutes les illustrations sont de l'auteur.

1. Suivant en cela L. Prieto (1975 : 101), j'emploie l'adjectif « sémiologique » pour désigner ce qui relève de la sémiologie, et l'adjectif « sémiotique » pour nommer ce qui relève de l'objet de la sémiologie.

2. Les significations dont sont porteurs les costumes populaires découlent généralement du chevauchement d'un système indicateur (constitué d'indices), d'un système sémiotique (constitué de signes) et d'un système symbolique, trois systèmes dont les limites sont souvent imprécises et fluctuantes. C'est à l'intersection des deux derniers que se situe le symbole saussurien, défini comme un signe motivé, c'est-à-dire fondé sur un lien analogique entre signifiant et signifié (Saussure 1968 : 101). L'usage anthropologique qui est fait ici de la notion de symbolisme conduit à l'étendre aux cas où la motivation possède un caractère homologique (dans un système comprenant au moins deux signes, les signifiants sont entre eux dans le même rapport que les signifiés).

3. Des guillemets 'simples' désignent les signifiés, des barres obliques les/signifiants/.

4. Sur la distinction entre unités de première et de seconde articulation, voir en linguistique A. Martinet (1967) et en sémiologie L. Prieto (1968).

5. Autrement dit, le processus de désémantisation présente deux stades : dans une première étape, l'ordonnance des couleurs, si elle n'est plus intégrée à un système symbolique conserve sa fonction sémiotique ; dans une seconde étape, la fonction sémiotique disparaît à son tour, les différences de forme n'étant plus rattachées à de quelconques signifiés. Ces deux étapes sont distinguées dans la figure 5.

6. Les costumes liés au veuvage introduisent sans doute une combinatoire, mais celle-ci, effective au plan de l'analyse formelle du vêtement en traits distinctifs, apparaît peu pertinente dans le processus de communication. Intervient en effet ici le système indicateur constitué par l'apparence physique du porteur du costume: ce qui permet de reconnaître une femme mariée d'un veuf, c'est moins le fait qu'une même coiffure rouge à bandes bleues et un même col droit soient portés en association avec des éléments différents (présence ou absence d'une bande décorative dans le bas de la tunique, etc.) que le fait qu'ils soient portés respectivement par une femme et par un homme.

7. Si ces deux traits vont toujours de pair dans les costumes analysés ici, l'existence d'unités de seconde articulation n'implique évidemment pas en droit l'homologie.

\section{RÉFÉRENCES}

Balfet, H. 1981 Construction de vêtements par fabrication du matériau, Vêtement et sociétés 1 : 81-90.

Bogatyrev, P. 1971 The Functions of folk costume in Moravian Slovakia. The Hague/Paris : Mouton.

Delaporte, Y. 1979 La Fonction sociale du signe dans les costumes populaires : le cas des ceintures à Kautokeino, Anthropos 74 (1-2) : 55-60.

— 1980 Le Vêtement lapon et la recherche scandinave, Études finno-ougriennes 14 : 115-134.

- 1981 Les Costumes du Nord de la Laponie, ou le degré zéro du signe, Vêtement et sociétés 1 : 323-333.

Martinet, A. 1967 Éléments de linguistique générale. Paris : A. Colin.

Prieto, L. 1968 La Sémiologie. In A. Martinet (dir.) Le Langage. Paris : Gallimard (Encyclopédie de la Pléiade) : 93-144.

— 1975 Pertinence et pratique. Paris : Éditions de Minuit.

Saussure (de), F. 1968 Cours de linguistique générale. Paris : Payot. 


\section{RÉSUMÉ}

Les Costumes du Sud de la Laponie : organisation et désorganisation d'un système symbolique. Une enquête dans les six provinces scandinaves correspondant au sud de la Laponie (Nordland, Nord-Tröndelag et Sör-Tröndelag en Norvège, Västerbotten, Jämtland et Härjedalen en Suède) a conduit à la découverte d'un système symbolique sous une forme organisée dans la commune de Grane (Nordland), où le costume est utilisé comme signifiant pour traduire les catégories de sexe et d'âge, les statuts de jeune fille et de femme mariée ainsi que plusieurs signifiés associés au deuil (concernant veuve et veuf depuis moins d'un an, veuve depuis plus d'un an, annonce d'un deuil). Ailleurs, ce système ne survit que sous la forme d'éléments isolés. L'analyse sémiologique des unités signifiantes composant le système organisé conduit à proposer une explication du maintien ou de la disparition de chacune d'elles dans le cadre du système désorganisé.

\section{ABSTRACT}

Southern Lapland Dress : the Organisation et Desorganisation of a Symbolic System. Thanks to a survied carried out in six scandinavian provincies (Nordland, Nord-Tröndelag and Sör-Tröndelag in Norway, Västerbotten, Jämtland and Härjedalen in Sweden) covering Southern Lapland, a symbolic system worns in native dress has been discovered. It has an organized form in Grane district (Nordland) were clothes are used as signifiers of the categories of sex and age, and of the status of girls and married women; there are also « signified », associated with mourning (widow or widower for less than a year, widow for more than a year). Elsewhere, only isolated elements of this system have survived. Owing to the semiological analysis of the signifier units that make up the organized system, the reasons that each of these units as been kept or abandonned in the disorganized system can be explained.

\section{MOTS CLÉS}

Laponie, Norvège, Suède, vêtement, fonction sémiotique, pertinence.

\section{KEYWORDS}

Lapland, Norway, Sweden, clothing, semiotic function, pertinence.

\section{NOTES ET RÉFÉRENCES DE l'INTRODUCTION}

Delaporte, Y. 2004 Le Vêtement lapon. Formes, fonctions, évolution. Paris, Leuven, Peeters (Artique) \& Oslo, Instituttet for sammenlignende kulturforskning, [Publication remaniée de : 1990, thèse de doctorat d'État en ethnologie, Université Paris 1]. 